\title{
Correlation between cluster analyses of Salmonella strains isolated from diarrhetic patients in Kuwait and biofilm formation
}

\author{
A. Al-Mousawi ${ }^{1}$, A. Eissa ${ }^{1}$, F. Abu-Zant ${ }^{1}$, H. Drobiova ${ }^{1}$, \\ I. Al-Saif ${ }^{2} \&$ E. Al-Saleh ${ }^{1}$ \\ ${ }^{1}$ Microbiology Program, Department of Biological Sciences, \\ College of Science, Kuwait University, Kuwait \\ ${ }^{2}$ Department of Microbiology, Food Laboratories, \\ Public Health Laboratory, Ministry of Health, Kuwait
}

\begin{abstract}
Salmonella is a highly diverse group of strains that belong to the Enterobacteriaceae and can cause many infections, such as diarrhea, pyrexia and septicemia, in humans and animals. One important virulence factor is the ability to form biofilm. In the present study, the potential to form biofilms by Salmonella strains isolated from diarrhetic patients was investigated and correlated with the strain type. Isolated bacteria were identified by sequencing of 16S rDNA. The potential of Salmonella to form biofilms was determined using bioluminescence microbial cell viability assay. In addition, the metabolic fingerprints of Salmonella were determined using the Biolog system, following the manufacturer's instructions. Cluster analysis based on catabolic activity and $16 \mathrm{~S}$ rDNA of isolated strains showed the tendency of most Schwarzengrund $(66.7 \%-70 \%)$ and E5 strains (85\%) to cluster individually, which implied the high distinctive genetic background of Schwarzengrund and E5 strains. On the other hand, Heidelberg and Paratyphi strains were clustered among other stains, which reflected the genetic resemblance of these strains to other Salmonella strains. The biofilm studies showed the high potential of the majority of E6 $(60 \%)$ and Heidelberg $(66.7 \%)$ strains to form biofilms, while low potential to form biofilms was displayed by $78 \%$ of Schwarzengrund strains.

Keywords: Salmonella, biofilm, $16 \mathrm{~S}$ rDNA sequencing, metabolic fingerprint.
\end{abstract}




\section{Introduction}

The enterobacteria are a heterogeneous group of Gram-negative rods that naturally inhabit the intestine of humans and animals. Salmonella, which is one of the important genera belonging to the Enterobacteriaceae, can cause many infections such as diarrhea, pyrexia and septicemia in humans and animals (D'Aoust [2]). Salmonella is usually found in poultry, poultry salads, meat, meat products, raw milk, shell eggs, egg custards, improperly cooked mayonnaise, ice cream, and sauces (Uyttendaele et al. [16]; Foley and Lynne [3]). Thus, Salmonella is one of the most common types of foodborne illnesses reported causing food poisoning or salmonellosis (D'Aoust [2]). Following the uptake of contaminated food or water, Salmonellae reach the intestine, cross the epithelial barrier, activate the host signal transduction cascades and induce the formation of membrane ruffles localized at the contact point between the bacterium and host cell (Jones et al. [5]; Vazquez-Torres et al. [17] and Rescigno et al. [12]). Ultimately Salmonellae are taken up in large vacuoles. The symptoms of salmonellosis develop within 12-36 hours of eating food containing Salmonella. Symptoms include nausea with vomiting, abdominal cramps and diarrhea, which can be severe (Tsolis et al. [8]; Kingsley et al. [8]; Ohl and Miller [11]). In recent years problems associated with Salmonella have increased considerably, both in terms of occurrence and severity of cases of human salmonellosis (Jewes [4]; Robertson et al. [14]).

An important factor influencing the pathogenicity of Salmonella is its ability to adhere to the host's intestinal surfaces prior to invasion (Robertson et al. [13]). This interaction is thought to depend upon bacterial-like adhesins recognizing specific glycoconjugate receptors on host cell surfaces. The possession of active flagella combined with chemotaxis is also an important factor in the pathogenicity of Salmonellae (Khoramian et al. [7]; Jones et al. [6]). Thus, variability in genotypic or phenotypic surface adhesion-related characteristics of different subspecies is expected to affect the establishment of Salmonellae populations in different hosts (Robertson et al. [13]) and thus its ability to cause outbreaks. One mechanism for ensuring survival in the host might be the differential biofilm-forming potential within a natural Salmonellae population. Biofilm formation is the net result of multiple interacting molecular events (Robertson et al. [13]) and is most conveniently measured at the phenotypic level. Thus, studies correlating strain types, antibiotic resistance and potential to form biofilm are required.

Many authorities require discrimination between different strains based on their metabolic pathways. Additionally, several high-resolution molecular fingerprinting techniques have been used to reveal species and subspecies diversity and provide tools to follow the persistence of particular infections, to recognize new infections and to assess the efficacy of control measures (Cheah et al. [1]). Typing methods based on comparisons of whole genomic DNA, plasmid DNA or specific genetic determinants have been used as supplementary techniques. Therefore, the aim of this work was to assess the ability of 
Salmonella strains to form biofilm and the correlation of biofilm formation potential with the metabolic and the genetic background of the different strains.

\section{Materials and methods}

\subsection{Sampling and initial identification of Salmonella}

Salmonella strains were isolated from stool samples brought from diarrhetic patients to the public health laboratory, Ministry of Health. For the isolation of Salmonella from stools, stool samples were inoculated into selinite broth to enrich for Salmonella. After overnight incubation, loop-full from selinite broth were streaked onto Hektoen enteric agar and incubated at $37^{\circ} \mathrm{C}$ for 48 hours (Vernacchio et al. [18]). Suspected grown Salmonella colonies were inoculated into sterile Triple Sugar Iron (TSI) agar slants, incubated at $35^{\circ} \mathrm{C}$ for $24 \mathrm{hrs}$ and Salmonella was identified following the Bacteriological Analytical Manual of the U.S. Food and Drug Administration (http://www.foodinfonet.com/ publication/fdaBAM.htm). Identified Salmonella were stored in 15\% glycerol nutrient broth at $-40^{\circ} \mathrm{C}$.

\subsection{Molecular identification of Salmonella by $16 \mathrm{~S}$ sequencing}

Isolated bacteria were identified by sequencing of $16 \mathrm{~S}$ rDNA. For this purpose, genomic DNA was purified from pure bacterial cultures using the Wizard Genomic DNA purification kit as recommended by the manufacturer (Promega). The concentration of extracted DNA were quantified by fluorometry with a model TK 100 fluorometer (Hoefer Scientific Instruments) by using the extended assay protocol of the manufacturer and then stored at $-20^{\circ} \mathrm{C}$. Then, $16 \mathrm{~S}$ rDNA sequences were amplified from extracted DNA using $27 \mathrm{~F}$ (AGAGTTTGATC(AC)TGGCTCAG) and 1492R (ACGG(CT)TACCTTGTTA CGACTT) primers (Kuske et al. [9]). All reactions were carried out in $25 \mu \mathrm{l}$ volumes, containing $12.5 \mathrm{pmol}$ of each primer, $200 \mu \mathrm{M}$ of each deoxyribonucleoside triphosphate, $2.5 \mu$ l of 10x PCR buffer $(100 \mathrm{mM}$ Tris- $\mathrm{HCl}$, $15 \mathrm{mM} \mathrm{MgCl} 2,500 \mathrm{mM} \mathrm{KCl} ; \mathrm{pH} \mathrm{8.3)}$, and $0.5 \mathrm{U}$ of $\mathrm{Taq}$ DNA polymerase (Applied Biosystems, UK), increased to $25 \mu \mathrm{l}$ with sterile water. PCR was performed in a Thermocycler, GeneAmp (Applied Biosystems, UK) with the following thermocycling program: 5 minutes denaturation at $95^{\circ} \mathrm{C}$, followed by 30 cycles of 1 minute denaturation at $95^{\circ} \mathrm{C}, 1$ minute annealing at $55^{\circ} \mathrm{C}, 1$ minute extension at $72^{\circ} \mathrm{C}$, and a final extension step of 5 minutes at $72^{\circ} \mathrm{C}$. PCR products were visualized by electrophoresis in $2 \%(\mathrm{wt} / \mathrm{vol})$ agarose gels and with ethidium bromide $(0.5 \mu \mathrm{g} / \mathrm{ml})$ staining. Then, PCR products were used as templates for DNA sequencing reactions. The sequencing PCR conditions were the same as those described above. Amplified DNA was purified by using a QIAQUICK PCR cleanup kit (Qiagen, Inc.), and DNA concentrations were determined as mentioned previously. Approximately $100 \mathrm{ng}$ of $16 \mathrm{~S}$ rDNA will be used as a template in dye terminator cycle sequencing reactions (Applied Biosystems PRISM dye terminator cycle sequencing kit). The 16S rDNA sequences obtained 
were run against the data bases using the basic alignment search tool (BLAST) and assigned to recognized representatives of the main eubacterial lineages based on scores of $97 \%$ or higher.

\subsection{Biofilm formation by Salmonella}

The potential of Salmonella for forming biofilms was determined using bioluminescence microbial cell viability Assay (Promega). For this purpose, 100 $\mu \mathrm{L}$ of overnight bacterial cultures ( $0.5 \mathrm{McFarland}$ standard) were transferred to sterile polystyrene 96 well microplates followed by another $100 \mu \mathrm{L}$ of phosphate buffer ( $\mathrm{pH} 7$ ). Microplates were incubated at $37^{\circ} \mathrm{C}$ for $24 \mathrm{hrs}$. Then, plates were washed gently with sterile phosphate buffer; $100 \mu \mathrm{L}$ of phosphate buffer was added followed by the addition of bioluminescence reagent. Contents of the plates were mixed on an orbital shake, incubated at room temperature for five minutes and luminescence values were taken on a microplate luminescence detector LD 400C (Beckman Coulter, USA). Control wells containing the phosphate buffer without cells were run to obtain a value for background luminescence.

\subsection{Determination of metabolic fingerprinting of Salmonella}

The metabolic fingerprints of salmonella were determined using a Biolog system following the manufacturer's instructions (OmniLog ${ }^{\circledR}$ ID System).

\section{Results}

Three different approaches were used to analyze and cluster isolated Salmonella. The phylogenetic analysis of the $16 \mathrm{~S}$ sequences using 97\% similarity index demonstrated the presence of five main phylotypes (Figure 1). Each phylotype was composed of different strain types. However, cluster analysis based on the ability of isolates to utilize different organic substrates showed the presence of highly diverse metabolic potentials of isolated Salmonella (Figure 2). Furthermore, isolated Salmonella were segregated into three main groups based on their potentials to form biofilm: isolates with high potential to form biofilm (Table 1(a)), isolates with low potential to form biofilm (Table 1(b)) and isolates unable to form biofilm (Table 1(c)). Each group contained different strain types.

\section{Discussion}

Cluster analysis based on the catabolic activity (Figure 2) and 16S rDNA (Figure 1) of isolated strains showed the tendency of most Schwarzengrund $(66.7 \%-70 \%)$ and E5 strains (85\%) to clusters individually, which implied the high distinctive genetic background of Schwarzengrund and E5 strains. On the other hand, Heidelberg and Paratyphi strains were clustered among other strains, which reflected the genetic resemblance of these strains to other Salmonella 


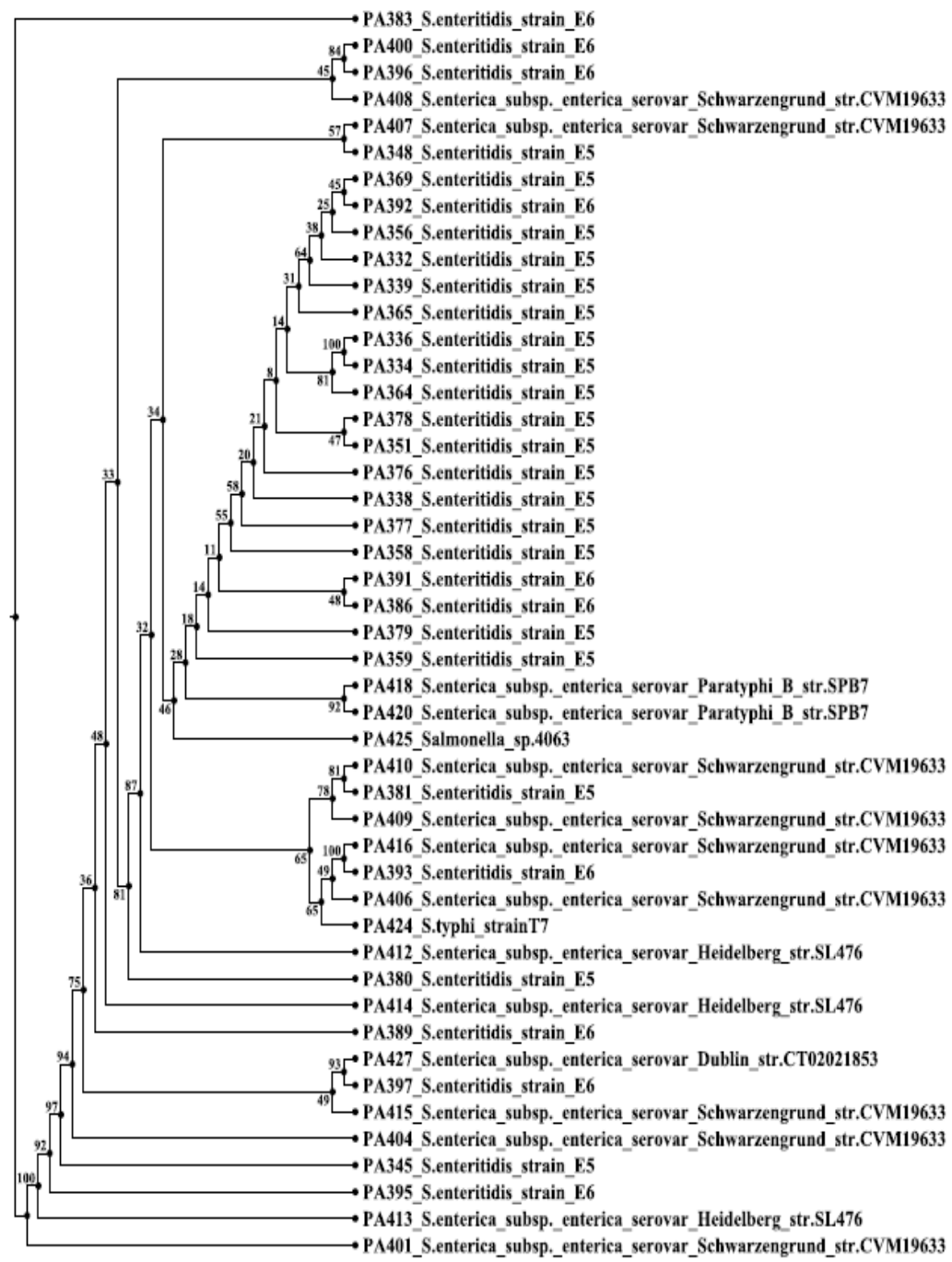

Figure 1: $\quad$ Phylogenetic tree of $16 \mathrm{~S}$ rRNA gene sequences clustered using the UPGMA method. The numbers at the nodes represent percentages of bootstrap sampling. 
72 Environmental Toxicology III

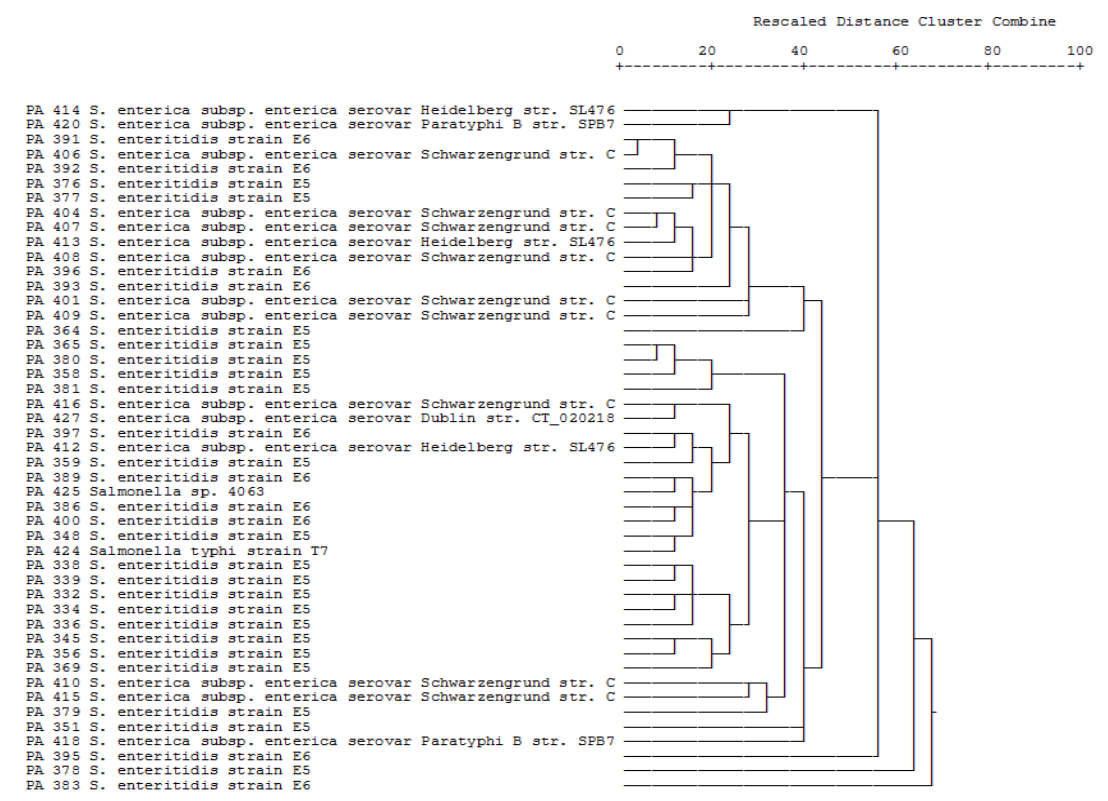

Figure 2: Cluster analysis tree of Salmonella based on metabolic fingerprinting obtained by Biolog.

Table 1: Biofilm formation by Salmonella strains. (a) Salmonella strains with high potential to form biofilm; (b) Salmonella strains with low potential to form biofilm; (c) Salmonella strains unable to form biofilm.

(a) Salmonella strains with high potential to form biofilm.

\begin{tabular}{|c|c|}
\hline Sample No. & Strain identity \\
\hline PA348 & S. enteritidis strain E5 \\
\hline PA339 & S. enteritidis strain E5 \\
\hline PA351 & S. enteritidis strain E5 \\
\hline PA338 & S. enteritidis strain E5 \\
\hline PA377 & S. enteritidis strain E5 \\
\hline PA345 & S. enteritidis strain E5 \\
\hline PA391 & S. enteritidis strain E6 \\
\hline PA393 & S. enteritidis strain E6 \\
\hline PA389 & S. enteritidis strain E6 \\
\hline PA397 & S. enteritidis strain E6 \\
\hline PA395 & S. enteritidis strain E6 \\
\hline PA383 & S. enteritidis strain E6 \\
\hline PA412 & S. enteric serovar Heidelberg \\
\hline PA413 & S. enteric serovar Heidelberg \\
\hline PA425 & Salmonella sp. 4063 \\
\hline
\end{tabular}


Table 1: $\quad$ Continued.

(b) Salmonella strains with low potential to form biofilm.

\begin{tabular}{|c|c|}
\hline Sample No. & Name of bacteria from BLAST \\
\hline PA369 & S. enteritidis strain E5 \\
\hline PA336 & S. enteritidis strain E5 \\
\hline PA334 & S. enteritidis strain E5 \\
\hline PA364 & S. enteritidis strain E5 \\
\hline PA358 & S. enteritidis strain E5 \\
\hline PA379 & S. enteritidis strain E5 \\
\hline PA400 & S. enteritidis strain E6 \\
\hline PA396 & S. enteritidis strain E6 \\
\hline PA392 & S. enteritidis strain E6 \\
\hline PA415 & S. enteric serovar Schwarzengrund \\
\hline PA401 & S. enteric serovar Schwarzengrund \\
\hline PA407 & S. enteric serovar Schwarzengrund \\
\hline PA410 & S. enteric serovar Schwarzengrund \\
\hline PA418 & S. enteric serovar Paratyphi $B$ \\
\hline PA420 & S. enteric serovar Paratyphi $B$ \\
\hline PA424 & S. typhi strain T7 \\
\hline PA414 & S. enteric serovar Heidelberg \\
\hline PA427 & S. enteric serovar Dublin \\
\hline
\end{tabular}

(c) Salmonella strains unable to form biofilm.

\begin{tabular}{|c|c|}
\hline Sample No. & Name of bacteria from BLAST \\
\hline PA408 & S. enteric serovar Schwarzengrund \\
\hline PA409 & S. enteric serovar Schwarzengrund \\
\hline PA406 & S. enteric serovar Schwarzengrund \\
\hline PA404 & S. enteric serovar Schwarzengrund \\
\hline PA365 & S. enteritidis strain E5 \\
\hline PA378 & S. enteritidis strain E5 \\
\hline
\end{tabular}

strains. The biofilm studies (Table 1) showed that the majority of E6 $(60 \%$ Table 1 (a)) and Heidelberg (66.7\% - Table 1 (b)) strains were able to form biofilm with different potentials, while a very low potential to form biofilm was displayed by $78 \%$ of Schwarzengrund strains.

\section{Conclusions}

Some Salmonella strains demonstrated high potential to form biofilm while other strains showed low potential to form biofilm. Biofilm formation potential was not correlated with the metabolic or the genetic background of the tested strains.

\section{Acknowledgements}

I would to thank the College of Graduate Studies (CGS) and Research Administration- Kuwait University (grant no. YM06/09) for funding the current project. Many thanks to the Biotechnology Center (BTC) - College of Science, Kuwait University for the provision of the sequencing facility. 


\section{References}

[1] Cheah, Y. Salleh, N. Lee, L. Radu, S. Sukardi, S. and Simm J. Comparison of PCR fingerprinting techniques for the discrimination of Salmonella enterica subsp. enterica serovar Weltevreden isolated from indigenous vegetables in Malaysia. World Journal of Microbiology and Biotechnology, 24(3):327-335, 2008.

[2] D'Aoust, JY. Salmonella and the international food trade. Int J Food Microbiol., 24(1-2):11-31, 1994.

[3] Foley, SL. and Lynne, AM. Food animal-associated Salmonella challenged: pathogenicity and antimicrobial resistance. Journal of Animal Science, 86 (14):E1 73-87, 2008.

[4] Jewes, L. A. Antimicrobial therapy of non-typhi salmonella and shigella infection. Journal of Antimicrobial Chemotherapy, 19:557-560, 1987.

[5] Jones, BD. Ghori, N. Falkow, S. Salmonella typhimurium initiates murine infection by penetrating and destroying the specialized epithelial M cells of the Peyer's patches. Journal of Experimental Medicine, 180: 15-23, 1994.

[6] Jones, BD. Lee, CA. and Falkow, S. Invasion by Salmonella typhimurium is affected by the direction of flagellar rotation. Infect Immun., 60:24752480, 1992.

[7] Khoramian, T. Haryama, S. Kutsukake, K. and Pechere, JC. Effect of motility and chemotaxis on the invasion of Salmonella typhimurium into HeLa cells. Microbial Pathogene, 9:47-53, 1990.

[8] Kingsley, R. Baumler A. and Oelschlaeger \& Hacker, J. (eds). Salmonella interactions with professional phagocytes in bacterial invasion into eukaryotic cells. New York: Kluwer Academic/Plenum, pp.321-342, 2000.

[9] Kuske, C. R. Barns, S. M. and Buschm J. D. Diverse uncultivated bacterial groups from soils of the arid southwestern United States that are present in many geographical regions App. Environ. Microbiol. 63:3614-3621, 1997.

[10] Lauren, M. Junker and Cladrym J. High-throughput screens for smallmolecule inhibitors of Pseudomonas aeruginosa biofilm development. Antimicrobial Agents and Chemotherapy, 51(10):3582-3590, 2007.

[11] Ohl, M. E. \& Miller, S. I. Salmonella: a model for bacterial pathogenesis. Annu Rev Med, 52:259-274, 2001.

[12] Rescigno, M. Urbano, M. Valzasina, B. Francolini, M. Rotta, G. et al. Dendritic cells express tight junction proteins and penetrate gut epithelial monolayers to sample bacteria. Nat Immunol., 2: 361-367, 2001.

[13] Robertson, J. Grant, G. Allen, E. Woodward, M. Pusztal, A. and Flint, H. Adhesion of Salmonella enteerica var Enteritidis strains lacking fimbriae and flagella to rat ileal explants cultured at the air interface or suberged in tissue culture medium. Journal Medical Microbiology, 49:691-696, 2000.

[14] Robertson, J. McKenzie, N. Duncan, M. Vercoe, E. Woodward, M. Flint, A. and Grant, G. Lack of flagella disadvantages Salmonella enterica serovar Enteritidis during the early staged of infection in the rat. Journal of Medical Microbiology, 52:91-99, 2003. 
[15] Tsolis, R. Kingsley, R. Townsend, S. Ficht, T. Adams, L. \& Baumler A. Of mice, calves, and men. Comparison of the mouse typhoid model with other Salmonella infections. Adv Exp Med Biol, 473:261-274, 1999.

[16] Uyttendaele, MR. Debevere, JM. Lips, RM. Neyts, KD. Prevalence of Salmonella in poultry carcasses and their products in Belgium. Int $J$ Food Microbiol., 40:1-8, 1998.

[17] Vazquez-Torres, A. Jones-Carson, J. Baumler, AJ. Falkow, S. Valdivia, R. et al. Extraintestinal dissemination of Salmonella by CD18-expressing phagocytes. Nature, 401: 804-808, 1999.

[18] Vernacchio, V. Vezin, R. Mitchel, A. Lesk, S. Plaut, A. Acheson, D. Characteristics of Persistent Diarrhea in a Community-Based Cohort of Young US Children. Journal of Pediatric Gastroenterology and Nutrition, 43(1): 52-58, 2006.

[19] United States Food and Drug Administration (FDA) http://www.foodinfonet.com/publication/fdaBAM.htm 